\title{
PROPUESTA PARA EL DESARROLLO DE LA LECTOESCRITURA ACADÉMICA COLABORATIVA MEDIADA POR PROYECTO WEBQUEST-WIKI
}

\author{
Kalinka Velasco Zárate ${ }^{1}$ \\ José Manuel Meza Cano² \\ Elsa Ma. Blancas Moreno ${ }^{3}$
}

\section{RESUMEN}

En este artículo, presentamos el diseño de un curso mixto y resultados de su implementación, donde un proyecto WebQuest-Wiki media el desarrollo de las habilidades de escritura colaborativa, en los docentes de idiomas en formación. El objetivo del diseño es promover el desarrollo de la alfabetización digital, del metadiscurso y la metacognición, a través de la tarea de escribir colaborativamente un artículo de investigación documental sobre un tema de su interés en su área académica. Las observaciones de las acciones, estrategias y procesos, junto con el cuestionario de evaluación del curso, permitieron estimar que la actividad WebQuest fue motivante y de utilidad para lectura y las habilidades digitales, seguida de la actividad Wiki, que permitió la práctica colaborativa de la escritura. Este tipo de diseño es útil para que los estudiantes realicen una tarea auténtica de investigación y lectoescritura colaborativa, que amplía sus conocimientos y disposiciones para el trabajo académico.

Palabras clave: WebQuest-Wiki. Alfabetización digital. Metadiscurso. Colaboración. Metacognición.

\section{Como citar este documento - ABNT}

VELASCO ZÁRATE, Kalinka; MEZA CANO, José Manuel; BLANCAS MORENO, Elsa Ma. Propuesta para el desarrollo de la lectoescritura académica colaborativa mediada por proyecto WebQuest-Wiki. Revista Docência do Ensino Superior, Belo Horizonte, v. 9, e012386, p. 1-19, 2019. DOI: https://doi.org/10.35699/2237-5864.2019.12386.

Recebido em: 06/04/2019 Aprovado em: 13/06/2019 Publicado em: 24/09/2019

\footnotetext{
${ }^{1}$ Universidad Autónoma Benito Juárez de Oaxaca, Oaxaca, México. ORCID ID: https://orcid.org/0000-0001-6610-3502. E-mail: kalivz@gmail.com.

2 Universidad Nacional Autónoma de México, Tlalnepantla, México.

ORCID ID: https://orcid.org/0000-0002-9504-7906. E-mail: manuel.meza@iztacala.unam.mx.

${ }^{3}$ Universidad Pedagógica Nacional, Oaxaca, México.

ORCID ID: https://orcid.org/0000-0002-9892-4193. E-mail: elsablancasm@gmail.com.
} 


\section{PROPOSTA PARA O DESENVOLVIMENTO DA LECTOESCRITURA ACADÊMICA COLABORATIVA MEDIADA PELO PROJETO WEBQUEST-WIKI}

\section{RESUMO}

Neste artigo, apresentamos o desenho de um curso misto e resultados de sua implementação, onde o projeto WebQuest-Wiki medeia o desenvolvimento das habilidades da escritura colaborativa nos docentes em formação de idiomas. O objetivo do desenho é promover o desenvolvimento da alfabetização digital, do metadiscurso e a metacognição, através da tarefa de escrever em colaboração um artigo de pesquisa documental sobre um tema de interesse na área acadêmica do grupo. As observações das ações, estratégias e processos, junto com o questionário de avaliação do curso, permitiram estimar que a atividade WebQuest foi motivante e de utilidade para leitura e as habilidades digitais, seguidas da atividade Wiki, que permitiu a prática colaborativa da escritura. Este tipo de desenho é útil para que os estudantes realizem uma tarefa autêntica de pesquisa e lectoescritura colaborativa, que amplia seus conhecimentos e suas disposições para o trabalho acadêmico.

Palavras-chave: WebQuest-Wiki. Alfabetização digital. Metadiscurso. Colaboração. Metacognição.

\section{PROPOSAL FOR THE DEVELOPMENT OF THE COLLABORATIVE ACADEMIC LITERACY MEDIATED BY WEBQUEST-WIKI PROJECT}

\section{ABSTRACT}

In this article, we present the design of a mixed course and results of its implementation, where a WebQuest-Wiki Project mediates the development of collaborative writing skills in the pre-service language teachers. The objective of the design is to promote the development of digital literacy, metadiscourse and metacognition, through the task of collaboratively writing a documentary research article about a topic of interest in their academic area. The observations of the actions, strategies and processes, together with the course evaluation questionnaire, allowed estimating that the WebQuest activity was motivating and useful for reading and the digital skills, followed by the Wiki activity, which allowed for the collaborative practice of writing. This type of design promotes the students' engagement into an authentic research and collaborative writing task, which increases their knowledge and their dispositions for the academic work.

Keywords: WebQuest-Wiki. Digital literacy. Metadiscourse. Collaboration. Metacognition. 


\section{INTRODUCCIÓN}

El propósito del artículo es presentar el diseño instruccional para un curso llamado Revisión de Textos Científicos, donde actividades didácticas presenciales basadas en el trabajo por Proyecto y en línea de la Web 2.0 como la WebQuest-Wiki median el desarrollo de la lectoescritura académica en un grupo de estudiantes universitarios, docentes de idiomas en formación. El diseño instruccional y su implementación tienen como meta que los estudiantes se vean inmersos en los procesos de interacción, colaboración, indagación y reflexión necesarios, tanto para la lectura, análisis y comprensión como para la escritura colaborativa de los textos académicos del llamado género de textos científicos, durante su formación profesional. La importancia de que los docentes de idiomas en formación obtengan conocimientos, habilidades y disposiciones para la lectoescritura de este tipo de textos reside en que estos son típicamente requeridos en proyectos de investigación en la universidad; de esta manera, los estudiantes universitarios acreditan en su capacidad para indagar un tema o problema del área de su especialidad, haciendo uso de la información o conocimiento generado por otros de manera reflexiva y creativa.

En general, para los estudiantes en el nivel licenciatura o de estudios superiores, la redacción en la lengua materna, L1, o en otra adicional, L2, de estos textos resulta una tarea compleja porque involucra conocimientos lingüísticos, procesos cognitivos, metacognitivos y de socialización aún por desarrollar (AGUILAR GONZÁLES; FREGOSO PERALTA, 2013; RÍOS NAVA; OLIVO ESTRADA, 2017). Por su parte, la enseñanza de la escritura académica en este nivel de estudios, generalmente sólo cubre la práctica de la redacción de textos descriptivos, de la redacción de párrafos y de temas de la búsqueda de información en fuentes documentales especializadas, de extracción y síntesis de la información en forma de notas con referencias bibliográficas aisladas y de resúmenes, llegando en pocas ocasiones a requerir de los estudiantes que las integren en un documento con un propósito y estructura específicos. Todavía menos frecuente, es que en los cursos de lectoescritura académica se llegue a conformar una experiencia de aprendizaje y práctica de lectoescritura con conciencia de los recursos y procesos que éstos involucran. Lo anterior plantea las siguientes interrogantes: ¿Qué recursos existen actualmente que puedan emplearse para el desarrollo de las habilidades de escritura académicas requeridas, ya sea en el idioma L1 u otro idioma meta?, ¿Cómo generar experiencias de la práctica de la lectoescritura de los textos científicos, en donde los intereses de los estudiantes, sus actuales formas de comunicación y de acceso a información multimodal, en medios como el internet sean integrados?, ¿Cómo propiciar que los estudiantes se apropien y transformen esta información multimodal de modo crítico? y ¿cómo garantizar que estos recursos sean aplicados a futuras tareas de la escritura académica? 
Para este caso, consideramos que el uso de la tecnología educativa de la Web 2.0 como la llamada WebQuest-Wiki (MARCH, 2007; KUTEEVA, 2011; HADJERROUIT, 2016; SALIDO LÓPEZ, 2015; GARCÍA BARRERA, 2015), acompañadas de una pedagogía apropiada, facilitan la comunicación, interacción, colaboración y reflexión, que los procesos de redacción de los textos del género científico requieren. Además, estas herramientas son una alternativa para mediar los procesos de construcción de estos conocimientos y habilidades, recuperando las experiencias y conocimientos previos del proceso de lectoescritura, en la lengua materna L1 y en la meta $L 2$, combinándolos con nuevos y más avanzados contenidos y habilidades como los de la alfabetización digital para el proceso de la escritura académica a nivel superior.

La organización del artículo es como sigue: primero, delineamos las diferentes perspectivas teóricas asumidas de la escritura académica y el papel de la tecnología educativa en propiciar su desarrollo, integrando lo que actualmente incluye el constructo de alfabetización digital (ADELL SEGURA, 2004; AREA MOREIRA; GUTIÉRREZ MARTÍN; VIDAL FERNÁNDEZ, 2012; AVELLO MARTÍNEZ et al., 2013). Luego, presentamos los recursos WebQuest-Wiki, sus características y usos en el aprendizaje y para la escritura académica. Segundo, describimos la situación diagnóstica del curso Revisión de Textos Científicos del programa de la Licenciatura en Enseñanza de Idiomas, Universidad Autónoma "Benito Juárez" de Oaxaca (UABJO), México, en el marco de un proyecto de investigación acción, que motivó la Propuesta de Diseño instruccional del curso mixto presentado y donde el trabajo por Proyecto mediado por WebQuest-Wiki tiene como propósito fomentar la metacognición y la autorregulación en el proceso de escritura académica colaborativa y desarrollar la alfabetización digital. Tercero, reportamos resultados de la evaluación de la experiencia y finalmente, ponderamos que el diseño instruccional Proyecto WebQuest-Wiki contribuye al desarrollo de las habilidades de la escritura académica colaborativa, entre otros procesos.

\section{ANTECEDENTES TEÓRICOS}

Las perspectivas teóricas asumidas para el diseño y el contenido del curso son, en primer lugar, las que reconocen la lectoescritura como una actividad lingüística y proceso en el que convergen los recursos lingüísticos y el potencial de las habilidades desarrolladas en experiencias previas en las prácticas de lectoescritura en la lengua materna L1 y las ya desarrolladas en la segunda L2 (COLOMBI; SCHLEPPEGRELL, 2002; FERREIRO, 2011); estas son necesarias para acceder e integrar nuevos conocimientos, habilidades y experiencias requeridos en la redacción de géneros académicos específicos, como los practicados generalmente por comunidades o grupos académicos especializados (LEMKE, 2002; AGUILAR GONZÁLEZ; FREGOSO PERALTA, 2013; ESPINO DATSIRA, 2015; RÍOS NAVA; OLIVO ESTRADA, 2017). Un concepto relacionado es el de Metadiscurso (HYLAND, 2004, 2005, 2009; KUTEEVA, 2011), es decir, la manera en la que, a través del uso de recursos interactivos e interpersonales, los escritores de textos académicos expresan ideas y hacen interpretaciones 
consistentes con su área disciplinar cubriendo las expectativas retóricas de esa comunidad académica en particular, considerando las habilidades de procesamiento de lector, sus recursos contextuales y sus experiencias intertextuales.

Los recursos interactivos conectan y relacionan ideas y párrafos en el texto; también guían al lector en la estructura y organización textual en el uso de las ideas de otros o la construcción de la intertextualidad. En cambio, los recursos interpersonales permiten al escritor de un texto académico construir su identidad como experto en el tema cuando argumenta, evalúa y opina sobre las ideas de otros, expresando así su actitud y posición respecto de éstas. Esto incluye el interpelar explícitamente al lector del texto, estableciendo así un lazo entre las ideas en el texto y las ideas que posiblemente el lector tenga sobre éste, en el entendido de que pertenecen a la misma comunidad de expertos; el empleo de los recursos interpersonales hace más evidente la relación dialéctica entre el escritor-lector en el texto (KUTEEVA, 2011).

Es necesario reconocer que, en la actualidad, las comunidades de especialistas que generan los textos en los que se comunican el resultado de actividades de investigación y de generación de conocimiento, hacen uso de recursos multimedia disponibles en la Web para tener acceso y difundir este tipo de textos, creando un vínculo intertextual entre los textos verbales y los multimedia (LEMKE, 2002; MORALES RUIZ et al., 2017). De esta manera, este entorno digital, novedoso y volátil, da paso a nuevas formas de comunicación, conformando una cultura escrita digital inédita:

[...] favorece la integración de los distintos sistemas de representación del conocimiento (habla, escritura, imagen estática y en movimiento, reproducción virtual, audio, etc.), en un único formato de manera que el texto adquiere la condición de multimedia (CASSANY, 2002, p. 4).

La integración de estos nuevos sistemas digitales de representación del conocimiento en los procesos de enseñanza y aprendizaje requiere destrezas de uso inteligente de las nuevas tecnologías y del dominio instrumental de las mismas para la búsqueda, análisis, selección, organización, aplicación, interacción y comunicación de datos e informaciones para que el alumno transforme la información en conocimiento (CASSANY, 2002; MORALES RUIZ et al., 2017). Es necesario saber apropiarse y otorgar significado a la densa información disponible en la Web, representada a través de múltiples lenguajes expresivos. Para Area, Gutiérrez \& Vidal (2012) y Avello Martínez et al., (2013), la apropiación del significado y expresión multimodal son nuevos términos para los conceptos tradicionales de leer y escribir en todo proceso alfabetizador. La colaboración, cooperación, comunicación efectiva y capacidad para compartir, crear y publicar contenidos resultan necesarios para el desarrollo y aplicación del pensamiento crítico, la creatividad, innovación y solución de problemas hacia la comprensión social y cultural (BEBEA GONZÁLES, 2015; FERREIRO, 2011). 
Actualmente, nos encontramos frente a jóvenes universitarios con conocimientos y habilidades previas de la alfabetización tradicional (FERREIRO, 1988, 2011), en español como L1 o L2, quienes también tienen conocimientos y experiencias previas del uso de la tecnología y de recursos digitales: computadoras con procesadores de texto, navegación en el internet, redes sociales y diferentes tipos de recursos multimedia y aplicaciones para uso diario y académico. Así, la pedagogía de la escritura y de los géneros discursivos académicos, puede integrar diferentes elementos del entorno digital que están transformando los procesos de escrituración con conciencia de los procesos que ésta conlleva (FERREIRO, 1988; GEE, 2005). De esta manera, el estudiante universitario, frente a la tarea de escribir textos académicos en su L1 $\circ$ L2, puede hacerlo empleando múltiples recursos disponibles, familiares u otros nuevos; así, los conocimientos del proceso de lectoescritura desarrollados en su idioma y los conocimientos y experiencias previas en los entornos digitales (MORALES RUIZ et al, 2017), sirven de umbral cognitivo o de andamiaje de su siguiente aprendizaje y posiblemente del aprendizaje de otros. También, el estudiante-escritor habrá de reconocer que el proceso de escrituración en cualquier idioma requiere de un propósito e involucra tanto al escritor como para quién se escribe; requiere desarrollar habilidades para la comprensión, ejercer la crítica y desarrollar la creatividad. Por último, el lenguaje requiere ser visualizado como un objeto susceptible de análisis desde escenarios mediados por la TIC (FERRERIRO, 2011; AVELLO MARTíNEZ et al., 2013).

Area Moreira, Gutiérrez Martín \& Vidal Fernández (2012) proponen situar el uso de las TIC para la alfabetización dentro de un modelo de práctica educativa que incorpora y entiende la alfabetización como el desarrollo de competencias audiovisuales y digitales, especialmente para que los estudiantes den significado al conocimiento que encuentran; cuestionar el libro de texto como fuente única del conocimiento y buscar otras; favorecer el método de aprendizaje basado en proyectos; generar actividades de expresión y comunicación empleando la tecnología, favoreciendo procesos presenciales y distantes de aprendizaje colaborativo; asumir el papel del docente como organizador y supervisor; considerar que la alfabetización es multimodal, en distintos medios, culturas y lenguajes; integrar el proceso de alfabetización digital al currículo de las materias, no aislarlo. Partiendo de lo anterior, es posible considerar como viables estrategias pedagógicas que integran actividades de la Web 2.0 como la WebQuest-Wiki, para mediar entre la lectoescritura tradicional, la alfabetización digital, y la metacognición en los estudiantes universitarios.

\section{WEBQUEST-WIKI}

Varios estudios sugieren que la implementación de actividades como las WebQuest-Wiki (MARCH, 2007; KUTEEVA, 2011; HADJERROUIT, 2016; SALIDO LÓPEZ, 2015; GARCÍA BARRERA, 2015), acompañadas de una pedagogía apropiada, facilitan procesos de comunicación, interacción, colaboración, reflexión y del manejo de contenidos específicos a 
un área académica, que los procesos de redacción de los textos del género científico requieren. Ambas actividades requieren de los estudiantes la expresión y comunicación a través de recursos tecnológicos, empleando diversos formatos hipertextuales, multimedia y audiovisuales para un propósito, además de otros procesos. Por un lado, la WebQuest, es esencialmente una tarea de investigación a partir de un interés auténtico, llevada a cabo a través de una estructura o andamio de aprendizaje e incluye las siguientes etapas (MARCH, 2007; ADELL SEGURA, 2004; SALIDO LÓPEZ, 2015): la introducción, motivadora y que presenta el problema expuesto y cómo se va a resolver; los objetivos, que dan a conocer las capacidades a alcanzar, la tarea que permite sintetizar, organizar, clasificar y transformar en conocimiento la información recogida de internet y en diferentes publicaciones científicas sobre el tema a estudiar; el proceso, que informa de una manera muy estructurada y clara los pasos para resolver la tarea y el resultado final (por ejem. una PowerPoint, un mapa conceptual, una creación artístico-plástica o audiovisual, etc.). De esta manera, se transita por un proceso de aprendizaje en el que la colaboración, comunicación, manejo e intercambio de información ayuda a ampliar los conocimientos.

Por otra parte, la Wiki propicia la escritura colaborativa sobre el tema investigado, al añadir y editar información más de una persona, incrementando así las oportunidades de obtención de nuevo conocimiento de manera participativa y activa (HADJERROUIT, 2016; KUTEEVA, 2011; INGHAM, 2013; STANLEY, 2013). La Wiki soporta recursos textuales escritos, visuales y auditivos (ej. links a páginas web, videos, imágenes); además, permite rastrear o identificar cambios para comparar versiones del texto, facilitando el análisis del proceso. Adell Segura (2004) y March (2007) atribuyen a la WebQuest-Wiki los siguientes beneficios: fomentan la integración y construcción de conocimiento por medio de la experiencia, la comunicación e interacción en línea, la reflexión, el trabajo en equipo para la resolución de problemas de la vida real o la respuesta auténtica a una pregunta y el empleo de materiales del entorno, aumentando así la motivación intrínseca; promueven procesos como comparación, elaboración, contraste de hipótesis, etc.; impulsan la adquisición y el desarrollo de la alfabetización digital. Como Del Moral \& Villalustre (2008), citado en García Barrera $(2015$, p. 3) comentan, esto facilita los procesos de metacognición y autorregulación en la redacción de los textos al proporcionar "oportunidades de observación y análisis de los resultados de sus acciones e interacciones grupales, que quedan visibles como parte del proceso-creativo formativo, produciendo un aprendizaje constructivo y reflexivo". Así también, se propicia que los estudiantes se desarrollen como agentes autónomos y activos de la construcción de su conocimiento, en lo individual y colectivo (SALAS SOTO, 2008; BOEKAERTS, 1999). 


\section{DISEÑO DEL CURSO}

\section{DIAGNÓSTICO SITUACIONAL DEL CURSO REVISIÓN DE TEXTOS CIENTÍFICOS}

El curso Revisión de Textos Científicos es parte del Plan Curricular de una Licenciatura en Enseñanza de Idiomas (lengua extranjera inglés, español y lenguas originarias), es el primero en el área de formación Ilamada Investigación (http://www.idiomas.uabjo.mx/media/23/2017/08/Plan_de_Estudios_Lic_Ense\%C3\%B1anz a_de_Idiomas_Final.pdf), tiene una duración de un semestre, con 2 sesiones presenciales a la semana de 1 hora y media, con 3 horas para asesorías. Este curso y los subsecuentes, Metodología de la Investigación, Proyecto de Titulación y Seminario de Titulación I y II buscan que los estudiantes adquieran las habilidades para la investigación de problemas en el área de su disciplina: La enseñanza y aprendizaje de idiomas. Otras materias del programa como Taller de Redacción Académica en Español y Géneros Académicos en Español, también tienen como propósito que desarrollen habilidades para la redacción de textos académicos en este idioma, por lo que al llegar al curso en cuestión, los estudiantes cuentan con elementos básicos del proceso de redacción de este tipo de género.

El Programa Indicativo del Curso Revisión de Textos Científicos plantea como propósito que el estudiante comprenda los siguiente:

[...] la estructura y propósito de diferentes textos científicos (ensayo, reporte, artículos) a través de lectura de ejemplos y la comprensión de características y estructuras propias de cada tipo textual que le permitan identificar aquellos que pueden sustentar un proyecto de investigación al analizarlos y estar familiarizados con éstos en el área de su disciplina y como base para desarrollar sus propios textos (UNIVERSIDAD AUTÓNOMA "BENITO JUÁREZ" DE OAXACA, 2015, p. 1).

Para tal efecto, las estrategias de enseñanza sugeridas son "la lectura activa de textos académicos y la escritura de estos mismos (UNIVERSIDAD AUTÓNOMA "BENITO JUÁREZ" DE OAXACA, 2015, p. 1)". Para el ejercicio de la escritura, se indica que se practiquen ambos: tanto "el proceso (ej. Notas, ideas, comentarios), así como el producto (ej. Paráfrasis, párrafos, discusión extendida como, por ejemplo, un breve resumen de un libro)" (UNIVERSIDAD AUTÓNOMA “BENITO JUÁREZ” DE OAXACA, 2015, p. 1). También, la meta final del curso mencionada en dicho documento es:

preparar al alumno para investigar y luego escribir (en inglés) el documento de graduación tal como: tesis, memorias de servicio, manual de procedimientos, etc.; considerando que la meta general es introducir al alumno al "acto" académico de hacer investigación bibliográfica y que produzca sus propios escritos basándose en esas investigaciones. 
No obstante, dada la problemática que representa el número limitado de horas presenciales, la gestión del aula con un grupo numeroso de 30 estudiantes y la necesidad de plantear estrategias de enseñanza que contribuyan a la integración y aplicación de los contenidos a una tarea con un propósito definido, en el semestre Febrero 2017/Julio 2017, se inició un proyecto de investigación acción (GRIFFE, 2012). La investigación acción permite resolver problemas específicos dentro de un programa educativo, en este caso, uno que forma docentes de idiomas y de problemas en el contexto del curso Revisión de Textos Científicos. Así, a través de ciclos de observación de la propia práctica docente, una profesora que imparte el curso, coautora de este artículo, se propuso realizar, para el Ciclo l, ajustes en los siguientes aspectos: la competencia a desarrollar en el curso, la estrategia didáctica con que ésta se desarrollaría, los procesos de interacción y comunicación y la concepción de la evaluación. Inicialmente, esto implicó un tránsito hacia un paradigma centrado en los aprendizajes, en el uso de recursos del aprendizaje mixto (SHARMA; BARRET, 2007; PEÑA MORENO; RODRÍGUEZ NIETO; PADILLA MONTEMAYOR, 2014) y en particular de la Web 2.0 Wiki, así como el énfasis en el tratamiento de la escritura académica desde la perspectiva del metadiscurso y de la escritura como proceso (VELASCO ZÁRATE, 2018).

En la evaluación del Ciclo I (Febrero 2017/Julio 2017), por medio de un Cuestionario de Evaluación del Curso, se obtuvo información en donde los estudiantes destacaron la utilidad del uso de la Wiki para la redacción colaborativa del reporte del proyecto, las características del recurso como la posibilidad de compartir el trabajo en un mismo punto, colaborar y comunicarse de manera asíncrona. También, algunos estudiantes la identificaron como un recurso para escribir colaborativamente en otros trabajos, y más importante, que piensan usar con sus propios estudiantes en el futuro. Entre las desventajas, apuntaron a sus propias dificultades para organizar el trabajo y comprometerse o involucrarse en la tarea, además de la falta de familiaridad con las herramientas de edición en la Wiki (VELASCO ZÁRATE, 2018).

Como resultado de la evaluación heterogénea (i.e. los estudiantes a la docente y el curso) y las observaciones de la docente a la implementación del plan en el Ciclo I, se consideró necesario realizar para el Ciclo II nuevos ajustes al curso: 1) incorporar la noción de diseño instruccional (ARSHAVSKIY, 2014; SALAS SOTO, 2008) para el curso en la modalidad de aprendizaje mixto, para lo que fue necesaria la colaboración con pares académicos del área de educación en línea y de escritura académica; 2) incluir actividades que apoyan a los estudiantes-escritores en el uso de recursos de la escritura que permiten expresar las ideas propias y su reacción a las ideas de otros, a través de la evaluación crítica o reflexión, siguiendo las convenciones de la comunidad académica que produce este tipo de textos; 3 ) aprovechar los espacios presenciales y en línea para potenciar la interacción y reflexión para la conciencia de los recursos y los procesos que la redacción colaborativa de los textos del género científico requiere, con la intención de fomentar procesos de autorregulación del proceso de enseñanza-aprendizaje en el que se ven inmersos tanto los estudiantes como la 
docente; 4) incluir actividades para el desarrollo de habilidades de investigación como la búsqueda, selección y manejo de información en línea que los textos científicos requieren. Resultado de este ejercicio es la siguiente propuesta para el desarrollo de la escritura académica colaborativa mediada por WebQuest-Wiki.

\section{PROPUESTA}

Curso Revisión de Textos Científicos (4ㅇs semestre, Febrero 2018-Junio 2018, 2 sesiones presenciales a la semana de 1 hora y media, con 3 horas para trabajo en línea en la plataforma Moodle http://www.idiomas.uabjo.mx/). La estrategia de trabajo propuesta es de proyecto mediado por WebQuest-Wiki, integrando las dimensiones de la metacognición y autorregulación de los procesos de aprendizaje, los recursos del metadiscurso para la escritura académica y de la alfabetización digital para el desarrollo de la siguiente competencia y micro-competencias (ver Tabla 1):

Elabora un reporte de investigación documental integrando en éste las características estructurales del género de este tipo de texto científico y los recursos del metadiscurso (interactivos e interpersonales) para la escritura académica, así como estrategias y habilidades digitales para la búsqueda y empleo de la información.

Micro-competencias

1. Identifica las características del género texto científico en artículos y reportes de investigación de temas relacionados con la enseñanza-aprendizaje de idiomas.

2. Emplea estrategias para la comprensión lectora de textos en L1 y la lengua meta así como estrategias metacognitivas para la autorregulación del aprendizaje.

3. Emplea medios digitales (internet, bases de datos, WebQuest, Wiki) para la ubicación, selección procesamiento y organización de información relevante en los textos.

4. Emplea estrategias de escritura académica (metadiscurso-interactivos e interpersonales) para la redacción y edición colaborativa de un reporte/artículo de investigación.

5. Comunica de manera oral en la lengua meta el contenido de la investigación documental realizada.

6. Evalúa las actividades, los medios, los procesos e interacciones durante el desarrollo de la investigación (propias, la de otros estudiantes y del curso).

Tabla 1 - Competencia y micro-competencias del curso

Fuente: Los autores.

Para el desarrollo de estas competencias se proponen tres unidades temáticas: Unidad 1: El género Textos Científicos, Unidad 2: Lectura de los textos y Unidad 3: El proceso de la escritura a lo largo de las cuales se realizarán actividades presenciales y en línea alojadas en 
el curso en la plataforma Moodle. Los temas y actividades del curso se muestran en la Tabla 2.

\begin{tabular}{|c|c|c|}
\hline Temas & Actividades presenciales & Actividades en línea \\
\hline $\begin{array}{l}\text { Género textos } \\
\text { científicos: Subgénero: } \\
\text { artículo o reporte de } \\
\text { investigación } \\
\text { - La metacognición y } \\
\text { elementos de ésta } \\
\text { indispensables para el } \\
\text { proceso de lecto- } \\
\text { escritura } \\
\text { - Las estrategias de } \\
\text { comprensión lectora } \\
\text { en la L1 y L2 } \\
\text { - Recursos del } \\
\text { Metadiscurso } \\
\text { - Herramientas digitales } \\
\text { de búsqueda, revisión } \\
\text { y selección de fuentes } \\
\text { para la investigación } \\
\text { del tema de interés. } \\
\text { - WebQuest-Wiki }\end{array}$ & $\begin{array}{l}\text { Discusiones en } \\
\text { asamblea } \\
\text { - } \text { Presentaciones } \\
\text { expositivas } \\
\text { - } \text { Lectura } \\
\text { colaborativa } \\
\text { - Uso de } \\
\text { organizadores } \\
\text { gráficos (tablas y } \\
\text { mapas) } \\
\text { - Ejercicios de } \\
\text { escritura }\end{array}$ & $\begin{array}{l}\text { - Foros } \\
\text { - Cuestionarios } \\
\text { - WebQuest; y uso de } \\
\text { Bases de datos (Acceso } \\
\text { Abierto) y Apps (DropBox, } \\
\text { MindDomo, Google Drive) } \\
\text { para la gestión de la } \\
\text { información y su } \\
\text { organización } \\
\text { - Wiki }\end{array}$ \\
\hline
\end{tabular}

Por medio de estas actividades se pretende desarrollar los contenidos propuestos y movilizar en los estudiantes sus conocimientos previos y habilidades para la organización y comunicación y disponerlos a la tarea de desarrollar el mini-proyecto colaborativo de investigación mediado por la WebQuest-Wiki, sobre un tema de interés en el área de la enseñanza-aprendizaje de idiomas a partir de la recuperación y reflexión de experiencias como estudiantes de idiomas y docentes en formación. La escritura del reporte/artículo escrito en la lengua meta inglés se aborda desde un enfoque de proceso, para fomentar el desarrollo de la metacognición y elementos como la autorregulación y autoeficacia para la escritura académica, a través de la observación y reflexión sobre las estrategias, recursos cognitivos, lingüísticos, interacción y aprendizaje en general.

La evaluación se propone como un proceso continuo formativo, en el que participan los estudiantes y la docente: 1) Fase diagnóstica del curso donde las competencias a desarrollar y las estrategias didácticas y de aprendizaje para lograrlo se presentan y donde los estudiantes identifican sus conocimientos previos sobre la lectoescritura y sus prácticas sociales y escolares. 2) Evaluación formativa sobre las actividades presenciales y en línea 
relacionadas con el desarrollo del Proyecto y la WebQuest-Wiki; incluye técnicas de evaluación por criterios (MOON, 2004), tanto del proceso como del producto, con retroalimentación derivada del diálogo y observaciones, sobre ambos procesos del proyecto y de la escrituración del texto, entre los estudiantes-escritores y estudiantes-docentes. De esta manera, la colaboración en el proyecto considera la retroalimentación de los involucrados sobre las acciones y sus resultados para la toma de decisiones hacia el logro de la meta, fomentando así la autorregulación (GARCÍA BARRERA, 2015). 3) Evaluación sumativa que requiere la socialización del reporte de investigación escrito por medio de una presentación oral, para la práctica de otra forma común del discurso académico. La Figura 1 es una línea del tiempo en donde se muestran la distribución de las unidades del curso propuesto.

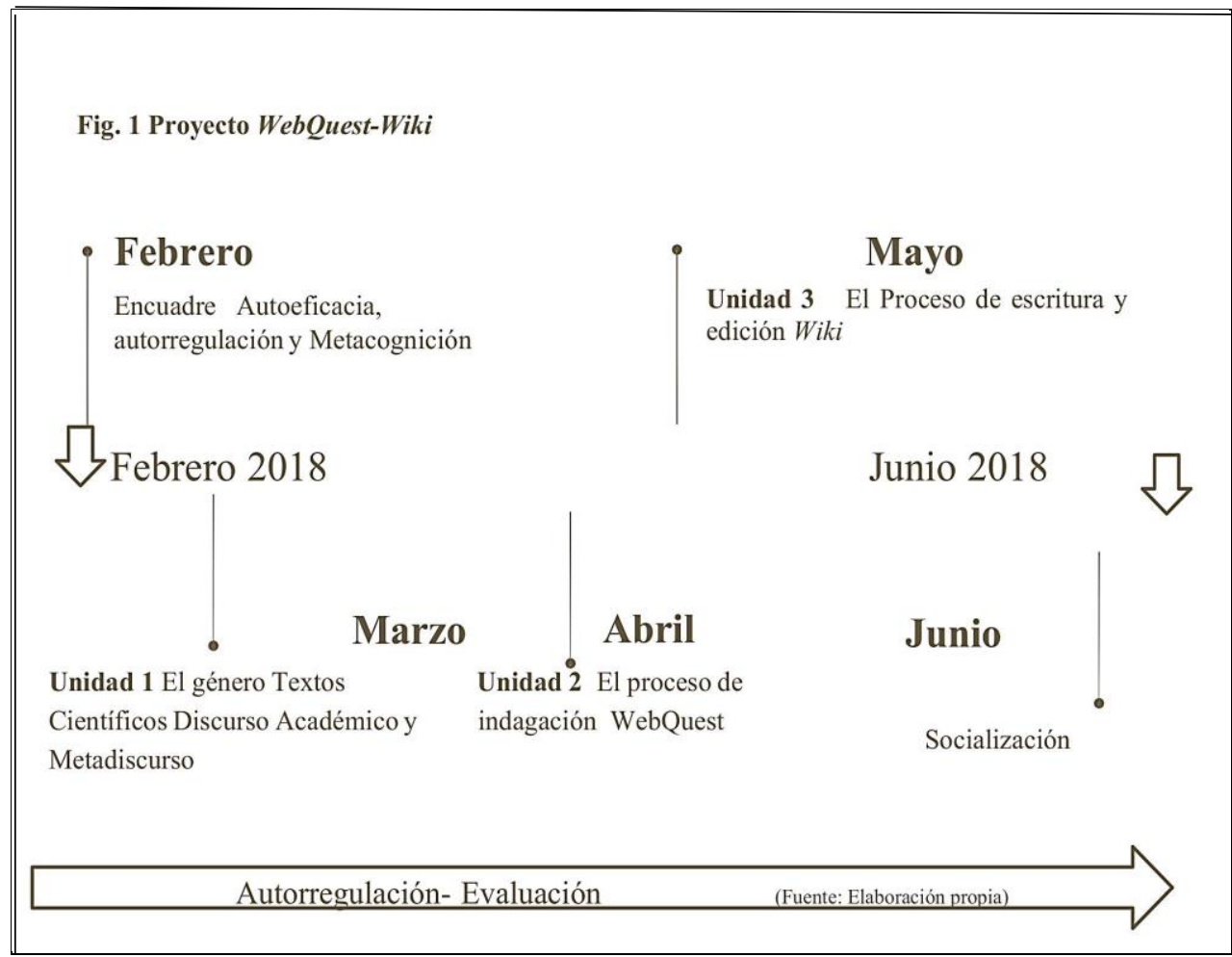

Figura 1 - Proyecto WebQuest-Wiki

Fuente: Los autores.

Se considera que la integración a la estrategia de aprendizaje por proyecto de la herramienta WebQuest-Wiki permite acercar al estudiante a una tarea auténtica que lo involucra cognitiva y motivacionalmente en un proyecto, planteando un objetivo claro a alcanzar, por lo que se espera que la implicación de los participantes en la actividad de indagar y redactar colaborativamente en la lengua meta sea alta, dado que responde directamente a sus intereses académicos. Además, se espera que los estudiantes transiten por el proceso de escrituración del tipo de texto redactado con más conciencia de las acciones, estrategias de aprendizaje, comunicación, evaluación, etc. los recursos empleados y logros obtenidos. 


\section{EVALUACIÓN DE LA EXPERIENCIA}

\section{Participantes:}

Participaron $\mathrm{N}=30$ docentes de idiomas en formación (19-26 años, L1 Español y lenguas originarias como el Mixteco y Zapoteco/ L2 Inglés Niveles A2, B1, Oxford Placement Test de Allan, 2001). Sin embargo, sólo se reportan datos cualitativos de $\mathrm{N}=26$ y cuantitativos de $\mathrm{N}=11$ estudiantes, quienes de manera voluntaria respondieron el Cuestionario Evaluación del Curso. Todos los participantes otorgaron consentimiento escrito para usar con fines de investigación la información derivada del proyecto, sin afectar con esto sus evaluaciones.

\section{Instrumentos:}

a) Hojas de retroalimentación y autoevaluación formativa

Durante el curso se obtuvieron comentarios de los estudiantes, registrados en hojas de autoevaluación y de evaluación del equipo ( 26 hojas de autoevaluación individual, 6 equipos autoevaluados), a modo de retroalimentación y como una estrategia para recuperar de forma escrita las fases, procesos, actividades y estrategias requeridas para la tarea que fue desarrollada de forma gradual. Estos comentarios permitieron conocer sus opiniones sobre el Proyecto WebQuest- Wiki, el trabajo en colaboración y el trabajo en plataforma.

b) Cuestionario Evaluación del Curso

La evaluación de las actividades del Proyecto WebQuest-Wiki se hizo al final usando un Cuestionario ad hoc en línea (41 ítems), que permite obtener información general personal y valorar en una escala Likert de 5 puntos (1 Total Desacuerdo y 5 Total Acuerdo) las actividades realizadas en el curso: WebQuest, Wiki, comprensión lectora, identificación de los recursos del metadiscurso para el tipo de texto, colaboración de los equipos, motivación e importancia atribuida a la actividad.

\section{RESULTADOS}

\section{Autoevaluación formativa}

La actividad WebQuest: permitió conocer los tipos de artículos y aquellos que son útiles para el trabajo de investigación; la elección del tema por los miembros del equipo resulta interesante, motivante y requiere la colaboración en la generación de ideas; propició el uso de bases de datos (Redalyc, Scholar Google, Scielo, DOAJ) para la búsqueda y selección de información haciendo uso de palabras clave y de recursos como Dropbox para almacenar y compartir documentos y notas. 
La actividad Wiki: es útil pues permitió redactar el texto a distancia, editar y comentar; ayuda a desarrollar habilidades como poder citar, parafrasear, identificar ideas, organizarlas en un texto con un propósito.

Los estudiantes recuperaron estrategias, procesos y acciones necesarias para elegir el tema, documentarse, organizar la información y redactar el texto requerido: la comunicación para seleccionar y justificar el tema de interés a partir de observaciones y experiencias comunes en el aprendizaje de idiomas, la lluvia de ideas y el generar preguntas que guían la investigación y búsqueda de información, establecer palabras clave, hacer búsquedas en fuentes confiables, seleccionar textos considerados relevantes, leer e identificar ideas importantes, extraer notas y organizar la información en un mapa mental, comentar y discutir para aclarar ideas, además de la capacitación recibida para hacer búsquedas efectivas en internet.

Como dificultades para el trabajo destacaron la falta de familiaridad con el ambiente en la plataforma (recursos y acciones necesarias para los envíos de archivos, participación en discusiones en foro) y tiempos de envío; el manejo de los tiempos establecidos para las actividades en cada fase del proyecto (relacionado a otras actividades escolares e individuales como el tiempo dedicado a empleos); la falta de experiencias de trabajo colaborativo en línea; el nivel percibido en el idioma L2 (inglés) que la redacción del texto requería y la falta de vocabulario, así como la organización del contenido del texto ante la participación de diferentes personas con opiniones distintas.

Eventualmente los 6 equipos redactaron los textos (Español L1, Inglés L2) y su contenido fue socializado en una presentación oral en L2 Inglés y discusión de los temas con el grupo al final del curso.

\section{Cuestionario Evaluación del Curso}

Los resultados en la Tabla 3 muestran que, la actividad WebQuest fue valorada como la más útil por los estudiantes (Med 4.45) para el desarrollo de habilidades digitales, como la búsqueda de textos de investigación e información, guiada por palabras clave y preguntas de investigación en bases de datos en línea sobre el tema seleccionado para el proyecto. También esta actividad propició la lectura de artículos en L1 y L2 y la extracción de notas, su almacenamiento y gestión en una nube (Dropbox). Sin embargo, la puntuación fue más baja para las Estrategias para la Comprensión Lectora en L2 (Med 3.88), relacionadas con la comprensión del vocabulario (uso de diccionario, inferir significados por el contexto e identificación de cognados) y el uso de la estructura y elementos gráficos del texto para predecir y comprender el contenido de los textos. 
Kalinka Velasco Zárate, José Manuel Meza Cano, Elsa Ma. Blancas Moreno

\begin{tabular}{|c|c|c|c|c|c|c|}
\hline N válido (por lista) & 11 & Min & Máx & Media & $\begin{array}{l}\text { Error } \\
\text { Estándar }\end{array}$ & $\begin{array}{l}\text { Desviación } \\
\text { Estándar }\end{array}$ \\
\hline Colaboración & 11 & 1.50 & 5.00 & 3.72 & 304 & 1.00 \\
\hline $\begin{array}{l}\text { Habilidades Comprensión } \\
\text { Lectura }\end{array}$ & 11 & 3.25 & 4.50 & 3.88 & .118 & .393 \\
\hline Wiki & 11 & 3.00 & 4.40 & 3.93 & .137 & .456 \\
\hline Metadiscurso & 11 & 3.33 & 4.67 & 3.95 & .135 & .447 \\
\hline Motivación & 11 & 3.29 & 5.00 & 4.14 & .151 & .503 \\
\hline WebQuest & 11 & 3.83 & 5.00 & 4.45 & .098 & .325 \\
\hline Tabl & uilo & $\begin{array}{l}\text { os del } \\
\text { Fuen }\end{array}$ & $\begin{array}{l}\text { estio } \\
\text { Los a }\end{array}$ & $\begin{array}{l}\text { io Evalu } \\
\text { res. }\end{array}$ & n del Curso & \\
\hline
\end{tabular}

Enseguida, fueron también valoradas favorablemente, las actividades que buscaron propiciar la práctica de recursos del Metadiscurso (Med 3.95) y la colaboración en el proceso de escritura en la actividad Wiki (Med. 3.93), en donde los estudiantes planearon y redactaron integrando recursos del Metadiscurso como estructura de párrafos, recursos lingüísticos para la cohesión, uso de referencias en el texto al incorporar ideas de autores en los párrafos y reaccionar a éstos; Wiki fomentó la lectura del borrador y la edición colaborativa del mismo. En este Ciclo II, se destaca que en la Wiki se insertaron imágenes en el texto, se usaron hiperlinks para organizar el contenido del texto en formato índice $y$, en menor medida, links hacia sitios externos con información (en sección referencias la URL del texto), lo que muestra que la actividad fomenta el uso de recursos multimedia en los textos. Además de propiciar la investigación y la lectoescritura académica, los docentes de idiomas en formación consideraron la WebQuest-Wiki relevante para su formación docente y para investigar temas de su área disciplinar, lo que reflejaron en la valoración de la dimensión Motivación (Med 4.14).

Aunque favorables, los resultados también apuntan a la necesidad de generar recursos de apoyo para que los estudiantes con poca experiencia en el trabajo en plataforma encuentren fácil y accesible su uso. Además, se requiere de actividades o estrategias para que los alumnos desarrollen habilidades de comunicación y negociación para la resolución de conflictos que surgen entre los miembros de los equipos durante la colaboración en diferentes etapas del proyecto. 


\section{CONCLUSIÓN}

La propuesta del curso en que se propone el desarrollo de habilidades de la escritura académica, la autorregulación y metacognición, a través del Proyecto WebQuest-Wiki, contribuyó a: familiarizar a los estudiantes con este tipo de diseños y herramientas; a la movilización de recursos cognitivos, de comunicación y actitudinales para desarrollar habilidades de la alfabetización digital que la preparación y escritura colaborativa de los textos académicos requiere. También se fomentó la metacognición de los recursos cognitivos y del lenguaje y de los procesos, estrategias y acciones requeridas para el logro del Proyecto; los estudiantes identificaron sus alcances y dificultades propias y del contexto en la práctica de la escritura académica, tanto en la L1 como en la L2. Finalmente, el incluir el diseño instruccional propuesto en el marco de un proceso de investigación acción permite la observación para realizar ajustes a las actividades y el análisis de distintos aspectos; un análisis por aparecer es la autoeficacia para la tarea específica y otro aspecto a analizar es el proceso de escrituración del texto y su relación con la autorregulación, que será abordado en otros reportes.

\section{REFERENCIAS}

ADELL SEGURA, Jordi. Internet en las aulas: Las WebQuest. Edutec: Revista Electrónica de Tecnología Educativa, n. 17, n. p., 2004. DOI: https://doi.org/10.21556/edutec.2004.17.530. Disponible en: http://www.cyta.com.ar/presentacion/mejora_archivos/edutec.htm. Acceso en: 17 marzo. 2017.

AGUILAR GONZÁLEZ, Luz Eugenia; FREGOSO PERALTA, Gilberto. La lectura de la polifonía e intertextualidad en el texto científico. Revista Mexicana de Investigación Educativa, v. 18, n. 57, p. 413-435, 2013. Disponible en: http://www.redalyc.org/articulo.oa?id=14025774005. Acceso en: 18 jul. 2017.

AREA MOREIRA, Manuel; GUTIÉRREZ MARTÍN, Alfonso; VIDAL FERNÁNDEZ, Fernando. Alfabetización digital y competencias informacionales. Madrid: Fundación Telefónica, 2012. Disponible en:

http://www.observatorioabaco.es/biblioteca/docs/147_FT_ALFABETIZACION_DIGITAL_2012 .pdf.

ARSHAVSKIY, Marina. Diseño Instruccional para Aprendizaje en Línea. U.S.A: Your ElearningWorld, 2014.

AVELLO MARTÍNEZ, Raidell; LÓPEZ FERNÁNDEZ, Raúl; CAÑEDO IGLESIAS, Manuel; ÁLVAREZ ACOSTA, Hugandy; GRANADOS ROMERO, John; OBANDO FREIRE, Francisco. Evolución de la alfabetización digital: nuevos conceptos y nuevas alfabetizaciones. MediSur, v. 11, n. 4, p. 450-457, 2013. Disponible en: http://scielo.sld.cu/scielo.php?script=sci_arttext\&pid=S1727897X2013000400009\&Ing=es\&tlng=es. Acceso en: 16 feb. 2018. 
BEBEA GONZÁLES, Inés. Manual de Alfabetización Digital Crítica. Una invitación a reflexionar y actuar. Madrid: BioCore, 2015.

BOEKAERTS, Monique. Self-regulated learning: where we are today. International Journal of Educational Research, v. 31, n. 6, p. 445-457, 1999.

CASSANY, Daniel. La alfabetización digital ALFAL. 2002. Disponible en: https://www.academia.edu/2976089/La_alfabetización_digital. Acceso en: 19 feb. 2018.

COLOMBI, Cecilia; SCHLEPPEGRELL, Mary J. Theory and Practice in the Development of Advanced Literacy. In: SCHLEPPEGRELL, Mary J.; COLOMBI, Cecilia (ed). Developing Advanced Literacy in First and Second Language. London: Lawrence Erlbaum Associates, 2002.

DEL MORAL, M. Ester; VILLALUSTRE, Lourdes. Las wikis vertebradoras del trabajo colaborativo universitario a través de WebQuest. Revista Latinoamericana de Tecnología Educativa, v. 7, n. 1, p. 73-83, 2008.

ESPINO DATSIRA, Sandra. La enseñanza de estrategias de escritura y comunicación de textos científicos y académicos a estudiantes de posgrado. RMIE, v. 20, n. 66, p. 959-976, 2015. Disponible en: http://www.comie.org.mx/documentos/rmie/v20/n066/pdf/66011.pdf. Acceso en: 19 julio. 2017.

FERREIRO, Emilia. Alfabetización: Teoría y práctica. D.F.: Siglo Veintiuno Editores, 1998.

FERREIRO, Emilia. Alfabetización digital: ¿De qué estamos hablando?. Educação e Pesquisa, v. 37, n. 2, p. 423-438, 2011. DOI: https://dx.doi.org/10.1590/S1517-97022011000200014. Disponible en: http://www.scielo.br/scielo.php?script=sci_arttext\&pid=S151797022011000200014. Acceso en: 20 feb. 2017.

GARCÍA BARRERA, Alba. Percepción de los estudiantes de magisterio acerca de la utilidad de las Wiki-Webquest en el aula tras su realización. Edutec. Revista electrónica de tecnología educativa. n. 54, p. 1-12, 2015. Disponible en: www.edutec.es/revista/index.php/edutece/.../Edutec_54_Garcia. Acceso en: 27 jul. 2017.

GEE, James, P. La ideología de los discursos. Lingüística social y alfabetizaciones. Madrid: Paidea y Morata, 2005.

GRIFFEE, Dale, T. An Introduction to Second Language Research Methods: design and data. eBook edition University of California, Berkeley: TESL-EJ Publications, 2012.

HADJERROUIT, Said. Assessing the Level of Collaborative Writing in a Wiki-based Environment: A Case study in Teacher Education. In: SPECTOR, Michael; IFENTHALER, Dirk; SAMPSON, Demetrios G.; ISAIAS, Pedro. Competencies in Teaching, Learning and Educational Leadership in the Digital Age (ed.). Papers from CELDA 2014. Basel: Springer International Publishing, 2016.

HYLAND, Ken. Disciplinary interactions: metadiscourse in L2 postgraduate writing. Journal of Second Language Writing v. 13, n. 2, p. 133-151, 2004. 
HYLAND, Ken. Patterns of engagement. Dialogic features and L2 undergraduate writing. In: RAVELLI, Louise; ELLIS, Robert (ed.). Analysing Academic Writing: Contextualized Frameworks. London: A\&C, Black, 2005.

HYLAND, Ken. Academic Discourse: English in a Global Context. London: Bloomsbury, 2009.

INGHAM, Louise. Using a Wiki to enhance the learning experience on a Business English Course. In: TOMLINSON, Brian; WHITTAKER, Claire (ed.). English Language Teaching: Course Design and Implementation. London: British Council, 2013.

KUTEEVA, María. Wikis and Academic Writing: Changing the writer-reader relationship. English for Specific Purposes, v. 30, n. 1, p. 44-57, jun. 2011.

LEMKE, Jay M. Multimedia Semiotics: Genres for Science Education and Scientific Literacy. In: SCHLEPPEGRELL, Mary J.; COLOMBI, Cecilia (ed). Developing Advanced Literacy in First and Second Language. London: Lawrence Erlbaum Associates, Publishers, 2002.

MARCH, Tom. Revisiting WebQuests in a Web 2 World. How developments in technology and pedagogy combine to scaffold personal learning. Interactive Educational Multimedia, $\mathrm{n}$. 15, p. 1-17, oct. 2007.

MOON, Jennifer. A Handbook of Reflective and Experiential Learning: Theory and Practice, London: Routledge Falmer, 2004.

MORALES RUIZ, Mario Ernesto; FLORES MACÍAS, Rosa del Carmen; MEZA CANO, JOSÉ MANUEL. El papel de las estrategias y los conocimientos previos al leer en Internet: revisión y discusión de la literatura relevante al tema. Avances en Psicología Latinoamericana, v. 35, n. 1, p. 131-141, 2017. Disponible en:

http://www.scielo.org.co/pdf/apl/v35n1/v35n1a10.pdf. Acceso en: 17 set. 2017.

PEÑA MORENO, José A.; RODRÍGUEZ NIETO, M. Concepción; PADILLA MONTEMAYOR, Victor M. Enfoques de concepciones de Aprendizaje Mixto, rol del profesor y estrategias de enseñanza de profesores de cursos mixtos. Revista Internacional de Ciencias Sociales y Humanidades, v. XXVI, n. 2, p. 179-198, jul/-dic. 2014.

UNIVERSIDAD AUTÓNOMA “BENITO JUÁREZ" DE OAXACA. Facultad de Idiomas. Programa Indicativo Curso Revisión de Textos Científicos, 2015. Disponible en:

http://www.idiomas.uabjo.mx/media/23/2017/08/Plan_de_Estudios_Lic_Ense\%C3\%B1anz a_de_Idiomas_Final.pdf2015. Acceso en: 16 feb. 2017.

RÍOS NAVA, Bernabé; OLIVO ESTRADA, J. Ramón. Los estudiantes de Medicina en la producción de textos académicos: Primeros pasos. In: REYES DE LA CRUZ, Virginia G.; ALVARADO JUÁREZ, Ana M. (coord.). La educación en México. Escenarios y desafíos. Ciudad de México: Juan Pablos Editor S. A., 2017.

SALAS SOTO, Silvia E. Diseño del Curso en Línea: Trabajo interdisciplinario. Revista Educación, v. 32, n. 1, p. 99-122, 2008. Disponible en: https://revistas.ucr.ac.cr/index.php/educacion/article/download/526/557/. Acceso en: 28 ene. 2018. 
SALIDO LÓPEZ. Pedro V. Nuevas Tecnologías, Universidad y Educación Artística: el diseño de WebQuest con rúbricas de evaluación en la formación de formadores. Edutec. Revista electrónica de tecnología educativa. n. 52, p. 1-10, jun. 2015. DOI: https://doi.org/10.21556/edutec.2015.52.308. Disponible en: https://www.edutec.es/revista/index.php/edutec-e/article/view/308. Acceso en: 17 jul. 2017.

SHARMA, Pete; BARRET, Barney. Blended Learning. Using Technology in and beyond the language classroom. Oxford: Macmillan, 2007.

STANLEY, Graham. Language Learning with Technology. Ideas for integrating technology in the classroom. Cambridge: Cambridge University Press, 2013.

VELASCO ZÁRATE, Kalinka. Escritura Académica Colaborativa: el uso de Wikis en un Programa de Licenciatura en Enseñanza de Idiomas. Edutec-e. Revista Electrónica de Tecnología Educativa, n. 63, p. 42-59, 2018. Disponible en: http://www.edutec.es/revista/index.php/edutec-e/article/view/969. Acceso en: 2 mayo 2018.

\section{Kalinka Velasco Zárate}

Facultad de Idiomas, UABJO. Doctora en Lingüística Aplicada, University of Essex, UK. Temas de interés: el papel de los factores internos (sistema lingüístico L1/L2, edad, procesos cognitivos) y su interacción con el contexto para explicar la variabilidad oral y escrita de la lengua meta; la lectoescritura académica colaborativa mediada por TICs.

kalivz@gmail.com

\section{José Manuel Meza Cano}

SUAyED FES Iztacala, UNAM. Doctor en Psicología de la misma institución con un proyecto sobre Entornos Personales de Aprendizaje y su influencia en el Aprendizaje Autorregulado y las Creencias Epistemológicas Específicas a internet. Sus investigaciones se enfocan al uso de internet para el aprendizaje y la docencia.

manuel.meza@iztacala.unam.mx

\section{Elsa Ma. Blancas Moreno}

Asesora académica de la Universidad Pedagógica Nacional, Unidad 201 Oaxaca. Doctora en Ciencias de la Educación, por el Instituto de Ciencias de la Educación de la UABJO. Temas de interés: Procesos educativos escolarizados vinculados con la desigualdad educativa (alfabetización en L2, comunicación en el aula) y las trayectorias educativas y los procesos de lectoescritura en el nivel superior. elsablancasm@gmail.com 